\title{
An Inverse Scattering Algorithm for the Segmentation of the Luminal Border on Intravascular Ultrasound Data
}

\author{
E. Gerardo Mendizabal-Ruiz ${ }^{1}$, George Biros ${ }^{2}$, and Ioannis A. Kakadiaris ${ }^{1}$ \\ 1 Computational Biomedicine Lab, Departments of Computer Science, Electrical and Computer \\ Engineering, and Biomedical Engineering, University of Houston, Houston, TX \\ 2 Department of Biomedical Engineering and School of Computational Science and \\ Engineering, Georgia Institute of Technology, Atlanta, GA
}

\begin{abstract}
Intravascular ultrasound (IVUS) is a catheter-based medical imaging technique that produces cross-sectional images of blood vessels and is particularly useful for studying atherosclerosis. In this paper, we present a novel method for segmentation of the luminal border on IVUS images using the radio frequency (RF) raw signal based on a scattering model and an inversion scheme. The scattering model is based on a random distribution of point scatterers in the vessel. The per-scatterer signal uses a differential backscatter cross-section coefficient (DBC) that depends on the tissue type. Segmentation requires two inversions: a calibration inversion and a reconstruction inversion. In the calibration step, we use a single manually segmented frame and then solve an inverse problem to recover the DBC for the lumen and vessel wall ( $\kappa^{l}$ and $\kappa^{w}$, respectively) and the width of the impulse signal $\sigma$. In the reconstruction step, we use the parameters from the calibration step to solve a new inverse problem: for each angle $\Theta_{i}$ of the IVUS data, we reconstruct the lumen-vessel wall interface. We evaluated our method using three $40 \mathrm{MHz}$ IVUS sequences by comparing with manual segmentations. Our preliminary results indicate that it is possible to segment the luminal border by solving an inverse problem using the IVUS RF raw signal with the scatterer model.
\end{abstract}

\section{Introduction}

Intravascular ultrasound (IVUS) is an invasive catheter imaging technique capable of providing high-resolution, cross-sectional images of the interior of human blood vessels. The IVUS catheter consists of a solid-state or mechanically-rotated transducer that emits ultrasound pulses and receives acoustic echoes (i.e., A-line) at a discrete set of angles (commonly 240 to 360). The envelopes of the received signals are computed, log-compressed, and then geometrically transformed to obtain the disc-shaped B-mode IVUS image.

Segmentation of IVUS images refers to the delineation of the lumen/intima and media/adventita borders. This process is necessary for assessing the vessel and plaque characteristics. Given that IVUS sequences may be hundreds to thousands of frames long, manual segmentation of a complete sequence is prohibitively time-consuming. Thus, methods for automatic segmentation of IVUS images are needed.

Contributions. In this paper, we present a novel method for segmentation of the luminal border on IVUS data. using the radio frequency (RF) raw signal based on a scattering

G.-Z. Yang et al. (Eds.): MICCAI 2009, Part II, LNCS 5762, pp. 885-892, 2009.

(C) Springer-Verlag Berlin Heidelberg 2009 
model and an inversion scheme. The main contribution of this work is a method for the segmentation that relies on a physics-based modeling of the IVUS signal instead of the IVUS B-mode images as in previous approaches. We evaluated our method using three $40 \mathrm{MHz}$ IVUS sequences by comparing the automatic segmentation result with manual segmentation. Our preliminary results indicate that it is possible to segment the luminal border by solving an inverse problem using the IVUS RF raw signal with a scatterer model.

Limitations. The model we present is quite simplistic both in the scattering approximation and in the spatial distribution of the scatterers. This is especially true in the lumen border. Also, shadow artifacts and side branches can create problems in the reconstruction.

Related work. Previous approaches for IVUS data analysis can be divided into two classes: image processing-based analysis and physics-based analysis. For the first class, the majority of methods relate to segmentation of the different layers of the vessel. Most reported successful approaches are based on contour detection by the minimization of a cost function. Recent proposed methods include those by Unal et al. [1] based on active shape models, Mendizabal-Ruiz et al. [2] using a probabilistic approach, Downe et al. [3] based on a 3-D graph search, and Papadogiorgaki et al. [4] based on wavelets. The input to all of the previous IVUS segmentation methods is the gray scale B-mode image. The limitation of these methods is a consequence of the fact that the appearance of the B-mode image depends on the characteristic of the IVUS system and the parameters used for the B-mode transformation. Thus, no segmentation method is guaranteed to perform correctly on IVUS images from different systems.

The second class of method relates to tissue classification from IVUS data. Although there are methods that work with the B-mode image [5]6], the most successful approaches are those focused on the characterization of atherosclerotic plaque composition by analysis of the ultrasound RF signal. Nair et al. [7] proposed a method known as "virtual histology" (IVUS-VH). Kawasaki et al. [8] proposed another method of tissue classification using the integrated backscatter (IB) parameter. O'Malley et al. and Katouzian et al. [9]10] explored methods for blood characterization. Mendizabal-Ruiz et al. [11] presented a method for the identification of contrast agent. However, none of these methods is designed for segmentation of the lumen/intima or media/adventitia.

\section{Methods}

For modeling the reflected IVUS signal, we chose to use the model employed by Rosales et al. [12]. This model assumes that the IVUS signal can be obtained from a physical model based on the transmission and reflection of ultrasound waves that radially penetrate the arterial structure. Since the wavelength produced by IVUS transducers is very large in comparison to the dimension of the structures of the vessel, this model assumes that structures can be modeled as a finite set of point scatterers with an associated differential backscattering cross-section coefficient (DBC). Although the signal in the transducer comes from a three-dimensional distribution of scatterers, in this paper we process the A-line scans independently and we consider two-dimensional distributions of scatterers. Consider an ultrasound pulse $P_{0}$ emitted at time $t_{0}$ with speed $c$ 


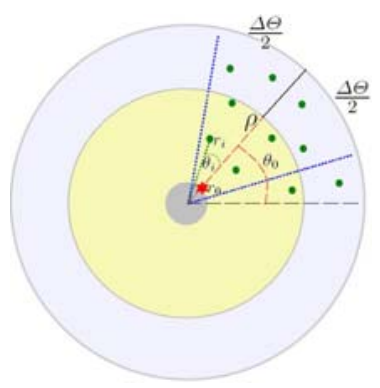

(a)

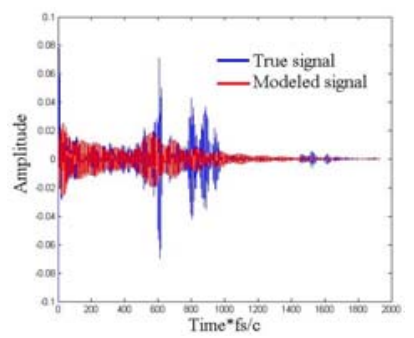

(b)

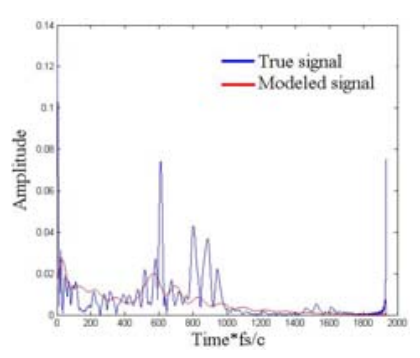

(c)

Fig. 1. (a) Scatterers interacting with the ultrasound beam on IVUS. (b) Raw real and modeled IVUS signals for a single angle. (c) Positive envelope of real and modeled IVUS signals for a single angle.

from the IVUS transducer with coordinates $\left(r_{0}, \theta_{0}\right)$, and that interacts at time $t_{i}$ with a scatterer located at a position $\left(r_{i}, \theta_{i}\right)$ with a DBC of $\kappa\left(r_{i}, \theta_{i}\right)$ (Fig. 1(a). The reflected pulse $P_{i}$ is a replica of the transmitted sound pulse $P_{0}$ that will return to the transducer at time $\left(t_{i}-t_{0}\right)$ and will be out of phase with respect to $P_{0}$ by $\frac{2 r_{i}}{c}$, where $c$ is the speed of sound in the medium. The ultrasound beam will interact with scatterers along its radial direction along an angular window given by $\Delta \Theta=\sin ^{-1}\left(1.22 \frac{\lambda}{D}\right)$ (Fig. 1(a), where $\lambda=\frac{c}{f}$ is the wavelength, $f$ is the transducer frequency and $D$ is the transducer diameter. Assuming Born approximation scattering, we use the principle of superposition to represent the total scattered wave as a sum of reflections from individual point scatterers [13]. Then, using this model, the ultrasound reflected signal for each transducer's angular position $\Theta_{k}$ at time $t$ for a finite set of $N$ scatterers with coordinates $\left(r_{i}, \theta_{i}\right)$ where $\theta_{i} \in\left\{\Theta_{k}-\frac{\Delta \Theta}{2}, \Theta_{k}+\frac{\Delta \Theta}{2}\right\}$ and $\mathrm{DBC} \kappa\left(r_{i}, \theta_{i}\right)$ is given by: $\hat{s}\left(t, \Theta_{k}\right)=C \sum_{i=1}^{N} \frac{\kappa\left(r_{i}, \theta_{i}\right) \exp \left(-\mu r_{i}\right)}{r_{i}} \exp \left(\frac{-\left(t-\frac{2 r_{i}}{c}\right)^{2}}{2 \sigma^{2}}\right) \sin \left(\omega t-\frac{2 r_{i}}{c}\right)$, where $\mu$ is the attenuation coefficient, $C$ defines the transducer constant parameters, and $\omega=2 \pi f$ is the angular velocity of the impulse function with width $\sigma$.

Recovering of the impulse signal width and the DBCs: The width of the impulse signal $\sigma$ is a parameter that depends on the characteristics of the particular IVUS transducer employed. However, since this parameter is not allays available, it is necessary to recover it from the IVUS data. Fontaine et al. [13 14] attempted to recover the scattering characteristics of blood, however, there is no consensus in the literature on the DBC values for blood. As we are mostly interested in reconstructing the lumen-wall interface, we use a two-step procedure that first calibrates scattering parameters and then inverts for the interface. We can therefore ignore the transducer-constant parameter $C$, since this is a constant and will only affect the scale of the resulting values. Additionally, we normalize the signal using the number of scatterers. Our modeled signal, then, is given by: $\hat{S}\left(t, \Theta_{k}\right)=\frac{1}{N} \sum_{i=1}^{N} \frac{\kappa\left(r_{i}, \theta_{i}\right) \exp \left(-\mu r_{i}\right)}{r_{i}} \exp \left(\frac{-\left(t-\frac{2 r_{i}}{c}\right)^{2}}{2 \sigma^{2}}\right) \sin \left(\omega t-\frac{2 r_{i}}{c}\right)$.

Specifically, our segmentation method requires two inversion steps: a calibration inversion (Algorithm 1) and a reconstruction inversion (Algorithm 2). Both steps employ 
the following assumptions: 1) there are only two layers within the vessel: lumen and vessel wall; 2) scatterers within the same layer will have the same DBC coefficient; 3 ) the attenuation coefficient $\mu$ is constant along the radial direction; and 4) the real IVUS signal can be approximated by a stochastic minimization process (Monte Carlo approach) on which we take random samples of the scatterers' positions.

If we know the radial position $\rho_{k}$ of the lumen border for a particular angle $\Theta_{k}$ from a manual segmentation, we can compute the width of the impulse signal $\sigma$ and the DBCs for lumen $\kappa^{l}$ and wall $\kappa^{w}$ by the minimization of the difference between the real IVUS signal $S\left(t, \Theta_{k}\right)$ and the signal computed with our model $\hat{S}\left(t, \Theta_{k}, \sigma, \kappa^{l}, \kappa^{w}\right)$. A significant difficulty is that we cannot treat the distribution of scatterers in a deterministic fashion. The scatterers' positions are the result of a spatial stochastic point process. Therefore, the minimization of the differences of the signals should be approached in a stochastic sense. There are many alternative methodologies for that purpose (e.g., stochastic optimization, Bayesian methods). In this paper, we consider the optimal parameter values as functions of the scatterer locations. Then, for each angle $k$ we generate $\xi$ samplings of scatterers' positions and minimize sum of the errors between the real IVUS signal and each of the $\xi$ modeled signals. Specifically, we solve the problem:

$$
\min _{\sigma_{k}, \kappa_{k}^{l}, \kappa_{k}^{w}} \frac{1}{2} \sum_{t} \sum_{i=1}^{\xi}\left(E\left(t, \Theta_{k}\right)-\hat{E}_{i}\left(t, \Theta_{k}, \sigma, \kappa^{l}, \kappa^{w}\right)\right)^{2},
$$

where $E\left(t, \Theta_{k}\right)$ and $\hat{E}_{i}\left(t, \Theta_{k}, \sigma, \kappa^{l}, \kappa^{w}\right)$ are the positive envelopes for the real and the modeled signals, respectively. Finally, we compute the median for each of the resulting parameters.

Segmentation: The radial position $\rho_{k}$ of the lumen border for each angle $\Theta_{k}$ can be recovered in a similar way. We use the parameters computed on the first inversion and we find $\rho_{k}$ by the minimization of the sum of differences between the real IVUS signal $S\left(t, \Theta_{k}\right)$ and the signals computed with our model $\hat{S}_{i}\left(t, \Theta_{k}, \rho_{k}\right)$ for each sampling $\xi$. Specifically, we solve:

$$
\min _{\rho_{k}} \frac{1}{2} \sum_{t} \sum_{i=1}^{\xi}\left(E\left(t, \Theta_{k}\right)-\hat{E}_{i}\left(t, \Theta_{k}, \rho_{k}\right)\right)^{2} .
$$

The sampling of the scatterers' positions is done by dividing the vessel into $P$ partitions, and on each partition we place a number of scatterers $N_{P}$ in random positions using a uniform distribution. The number of scatterers $N_{P}$ for each partition is determined by the area occupied by the partition and the density $\beta_{\alpha}$ (number of scatterers by unit area) corresponding to the layer $\alpha$ on which the partition is present.

The resulting curve might not be smooth due to noise. Moreover, artifacts (guidewire and shadows) and side branches may generate invalid points (outliers with respect to the curve points). Since we expect the number of invalid points to be small (these artifacts are present in small sections of the curve), we remove these outliers by applying clustering on the resulting curve points and eliminating the points corresponding to the smallest cluster. Finally, in order to constrain the curve to be smooth, we use an $\mathrm{L}_{1}$-minimization method combined with spectral smoothing [15] that also adds the property of periodicity to the curve. 


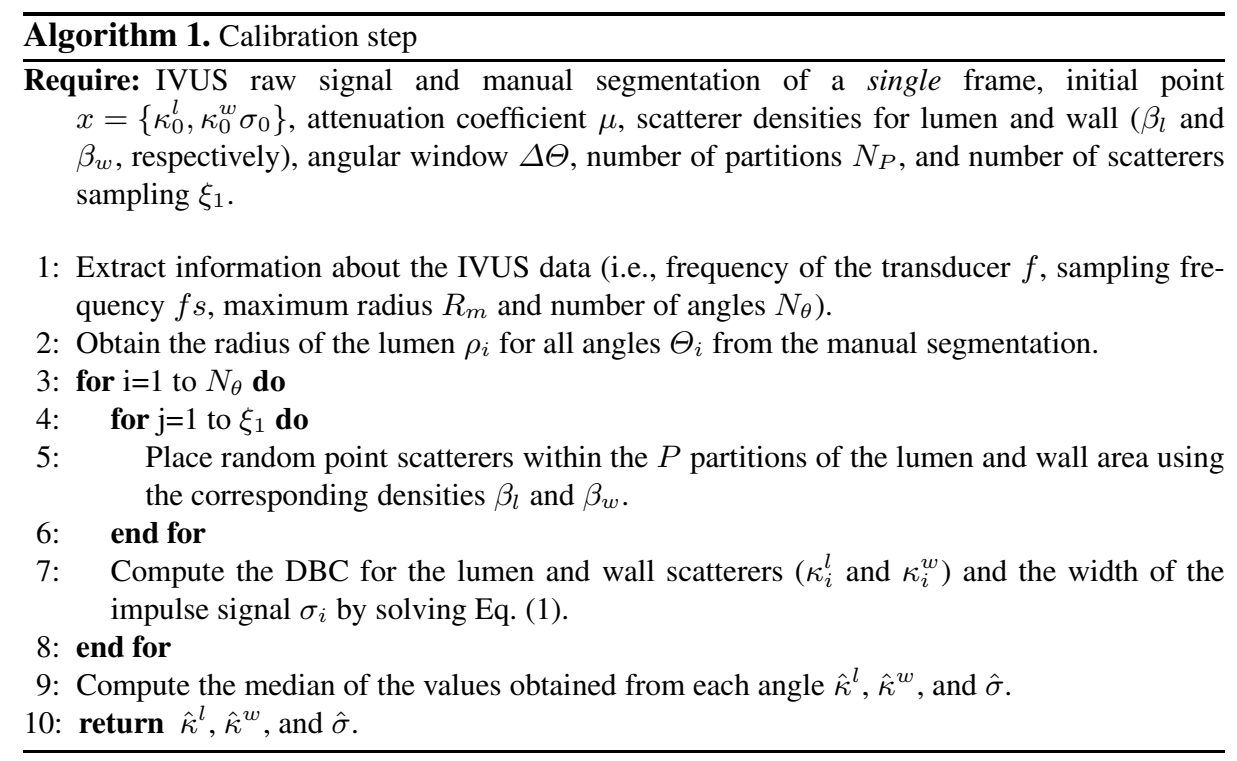

\section{Results}

We tested our method on 90 frames corresponding to three 40MHz IVUS sequences obtained from different rabbits' aortas. In order to be consistent with our first assumption, we ignored the section of the IVUS signal corresponding to the sheathing transducer (ringdown artifact). For the catheter used to acquired the data (Boston Scientific), the diameter of the transducer was approximately $0.9 \mathrm{~mm}$, while the angular window that we used for generating results was $\Delta \Theta=2.9^{\circ}$. Since our goal was to recover the lumen boundary, we used the typical attenuation coefficient for blood (i.e., $0.02 \mathrm{np} / \mathrm{cm}$ at $1 \mathrm{MHz}$ [ [16]. For our data frequency $(40 \mathrm{MHz})$, the attenuation coefficient $\mu$ corresponded to $0.8 \mathrm{np} / \mathrm{cm}$. The minimization was done using a simplex method. We used the voxel approach to create random scatterers [17]. Since in our experiments we had a radial resolution of $\delta_{r}=c / f=0.04 \mathrm{~mm}$, we set the voxel size to $V=16 \cdot 10^{-4} \mathrm{~mm}^{2}$. Experiments on syntectic data indicated that the exact value for the densities was not a determinant for the performance of our model provided $\beta_{l} \leq \beta_{w}$ (assuming a smaller density on lumen). Moreover, we chose the density values $\beta_{l}=219$ and $\beta_{w}=636$ according to the ratio of densities that we could expect on these tissues based on typical values for RBCs and epithelial cells [18]. The density used for the segmentation step was arbitrarily set constant to $\beta_{s}=1000$ for all the partitions $P$. The numbers of scatterer position samplings $\xi$ used for parameter recovering and lumen segmentation were $\xi_{1}=20$ and $\xi_{2}=10$, respectively.

The computed parameters $\left(\hat{\kappa}^{l}, \hat{\kappa}^{w}, \hat{\sigma}\right)$ for the first sequence were $\left(1.19 \cdot 10^{-9}, 3.54\right.$. $\left.10^{-9}, 6.33 \cdot 10^{-8}\right)$, for the second sequence were $\left(1.28 \cdot 10^{-9}, 2.77 \cdot 10^{-9}, 6.94 \cdot 10^{-8}\right)$ and for the third sequence were $\left(1.03 \cdot 10^{-9}, 2.84 \cdot 10^{-9}, 5.29 \cdot 10^{-8}\right)$. Figures $1(\mathrm{~b}, \mathrm{c})$ depict an example of a real IVUS signal and the adjusted signal using our model respectively. Figure 2 depicts examples of segmentation results. The segmentation results on the 90 

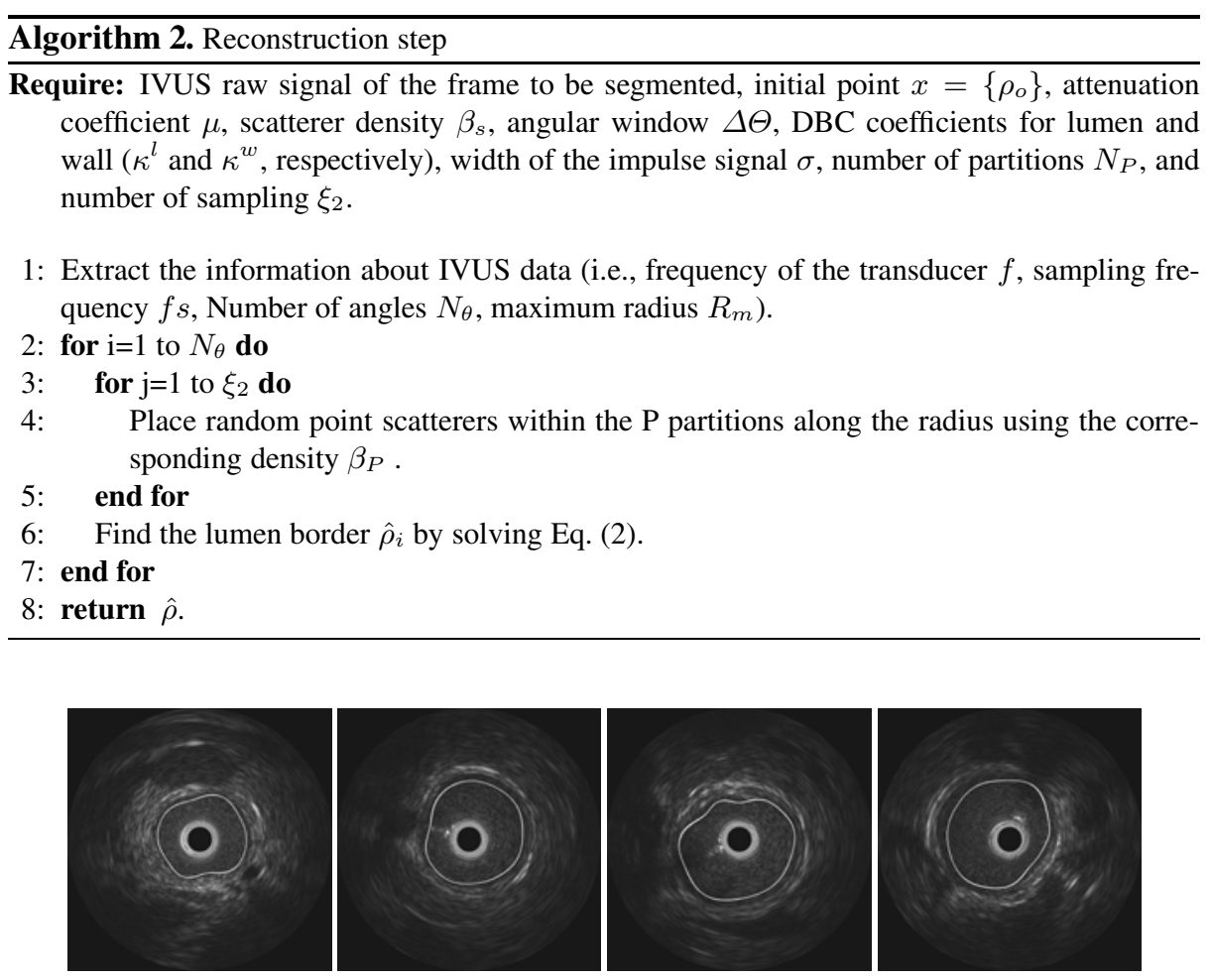

Fig. 2. Examples of segmentation results

frames were evaluated by comparing the agreement between areas corresponding to lumen on each frame by our method (A) with manual segmentations from two expert observers $\left(\mathrm{O}_{1}\right.$ and $\left.\mathrm{O}_{2}\right)$. The mean Dice similarity coefficient was $s=90.27$. In addition, we performed linear regression and Bland-Altman analysis, for which we report the inter-observer and automatic mean biases (mean area difference) and variabilities. The coefficient of determination ( $R^{2}$, where $R$ is the linear correlation) for area differences between $\mathrm{O}_{1}$ and $\mathrm{O}_{2}\left(\mathrm{O}_{1}, \mathrm{O}_{2}\right)$ was $R^{2}=0.98$, and $R^{2}=0.93$ and $R^{2}=0.93$ for $\left(\mathrm{A}, \mathrm{O}_{1}\right)$ and $\left(\mathrm{A}, \mathrm{O}_{2}\right)$, respectively. The bias of the area differences for $\left(\mathrm{O}_{1}, \mathrm{O}_{2}\right)$ was $(1.80 \pm 0.93)$. $10^{5} \mathrm{~mm}^{2}$, for $\left(\mathrm{A}, \mathrm{O}_{1}\right)$ the bias was $(-5.80 \pm 3.16) \cdot 10^{5} \mathrm{~mm}^{2}$, and for $\left(\mathrm{A}, \mathrm{O}_{2}\right)$ was $(-3.99 \pm 2.71) \cdot 10^{5} \mathrm{~mm}^{2}$. Figure 3 depicts the results of this analysis.

\section{Discussion}

The IVUS RF signal may vary between different systems and even between different sequences, since a different IVUS catheter is used every time. However, due the fact that we calibrate our method for each sequence using a one-frame manual segmentation on the parameter's recovery step, our method can overcome this limitation. The model we use is quite simplistic both in the scattering approximation and in the spatial distribution of the scatterers. This is especially true in the lumen border resulting in a 


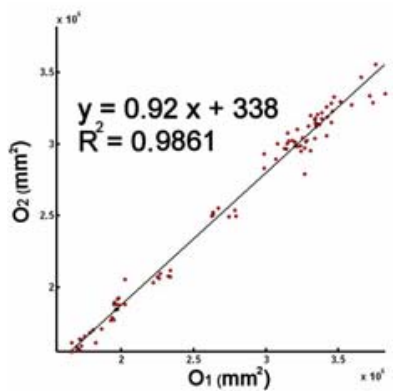

(a)

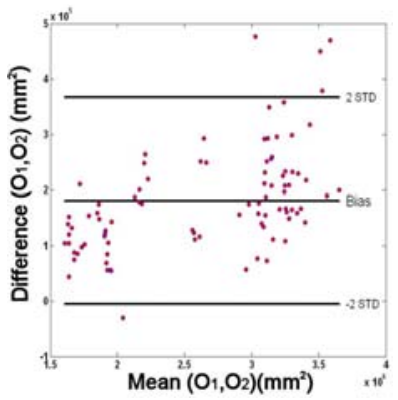

(d)

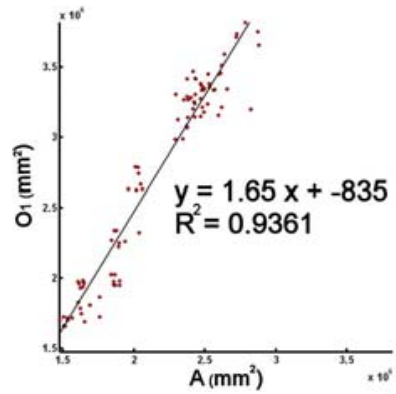

(b)

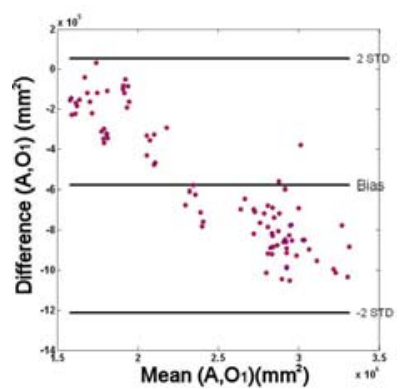

(e)

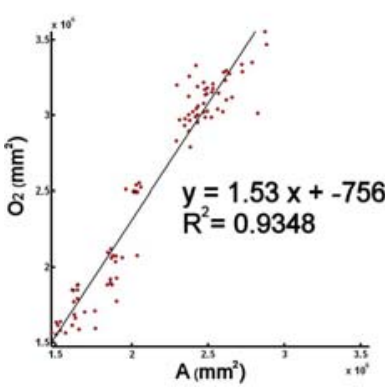

(c)

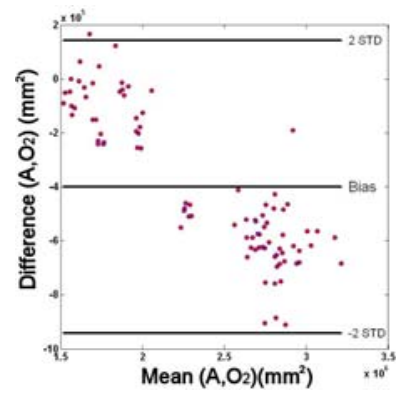

(f)

Fig. 3. Linear regression plot (a-c) and Bland-Altman plot (d-f) for $\mathrm{O}_{1}$ vs $\mathrm{O}_{2}(\mathrm{a}, \mathrm{d})$, A vs. $\mathrm{O}_{1}(\mathrm{~b}, \mathrm{e})$ and $\mathrm{A}$ vs. $\mathrm{O}_{2}(\mathrm{c}, \mathrm{f})$. Each point correspond to one of the 90 segmented frames.

under-segmentation by our method. Another limitation of our method relates to do with the time required to solve the inverse problems that limits the possibility of using the method on real time.

\section{Conclusion}

We have presented a novel method for segmentation of the luminal border on IVUS using a physics-based approach as opposed to an image-analysis based approach. To the best of our knowledge, this is the first method that segments IVUS images using the RF signal instead of B-mode images. Our preliminary results indicate that is possible to segment the luminal border by solving an inverse problem using the IVUS RF raw signal with a scatterer model. Future work includes the inclusion of additional layers, alternate methods for placing the scatterers, and comparison with image-based IVUS segmentation methods.

\section{References}

1. Unal, G., Bucher, S., Carlier, S., Slabaugh, G., Fang, T., Tanaka, K.: Shape-driven segmentation of the arterial wall in intravascular ultrasound images. IEEE Transactions on Information Technology in Biomedicine 12(3), 335-347 (2008) 
2. Mendizabal-Ruiz, E.G., Rivera, M., Kakadiaris, I.A.: A probabilistic segmentation method for the identification of luminal borders in intravascular ultrasound images. In: Proc. IEEE Conference on Computer Vision and Pattern Recognition, Anchorage, AK, Jun. 2008, pp. 18 (2008)

3. Downe, R., Wahle, A., Kovarnik, T., Skalicka, H., Lopez, J., Horak, J., Sonka, M.: Segmentation of intravascular ultrasound images using graph search and a novel cost function. In: Proc. $2^{\text {nd }}$ MICCAI Workshop on Computer Vision for Intravascular and Intracardiac Imaging, New York, September 10, 2008, pp. 71-79 (2008)

4. Papadogiorgaki, M., Mezaris, V., Chatzizisis, Y., Giannoglou, G., Kompatsiaris, I.: Image analysis techniques for automated IVUS contour detection. Ultrasound in Medicine and Biology 34(9), 1482-1498 (2008)

5. Roodaki, A., Taki, A., Setarehdan, S.K., Navab, N.: Modified wavelet transform features for characterizing different plaque types in IVUS images; a feasibility study. In: Proc. $9^{t h}$ International Conference on Signal Processing, Beijing, China, October 2008, pp. 789-792 (2008)

6. Katouzian, A., Baseri, B., Konofagou, E.E., Laine, A.F.: An alternative approach to spectrum-based atherosclerotic plaque characterization techniques using intravascular ultrasound (IVUS) backscattered signals. In: Proc. $2^{\text {nd }}$ MICCAI Workshop on Computer Vision for Intravascular and Intracardiac Imaging, New York (2008)

7. Nair, A., Kuban, B., Tuzcu, E., Schoenhagen, P., Nissen, S., Vince, D.: Coronary plaque classification with intravascular ultrasound radiofrequency data analysis. Circulation 106(17), 2200-2206 (2002)

8. Kawasaki, M., Takatsu, H., Noda, T., Sano, K., Ito, Y., Hayakawa, K., Tsuchiya, K., Arai, M., Nishigaki, K., Takemura, G., Minatoguchi, S., Fujiwara, T., Fujiwara, H.: In vivo quantitative tissue characterization of human coronary arterial plaques by use of integrated backscatter intravascular ultrasound and comparison with angioscopic findings. Circulation 105, 24872492 (2002)

9. O’Malley, S.M., Naghavi, M., Kakadiaris, I.A.: One-class acoustic characterization applied to blood detection in IVUS. In: Proc. Medical Image Computing and Computer-Assisted Intervention, Brisbane, Australia, pp. 202-209 (2007)

10. Katouzian, A., Baseri, B., Konofagou, E., Laine, A.: Automatic detection of blood versus non-blood regions on intravascular ultrasound (IVUS) images using wavelet packet signatures. In: Proc. Medical Imaging 2008: Ultrasonic Imaging and Signal Processing, San Diego, CA (Feburary 2008)

11. Mendizabal-Ruiz, E.G., Kakadiaris, I.A.: One-class acoustic characterization applied to contrast agent detection in IVUS. In: Proc. Medical Image Computing and Computer-Assisted Intervention Workshop on Computer Vision for Intravascular and Intracardiac Imaging, New York, September 10 (2008)

12. Ramirez, M., Ivanova, P., Mauri, J., Pujol, O.: Simulation model of intravascular ultrasound images. In: Proc. Medical Image Computing and Computer-Assisted Intervention, SaintMalo, France, pp. 200-207 (2004)

13. Fontaine, I., Bertrand, M., Cloutier, G.: A system-based approach to modeling the ultrasound signal backscattered by red blood cells. Biophysical Journal 77(5), 2387-2399 (1999)

14. Fontaine, I., Savery, D., Cloutier, G.: Simulation of ultrasound backscattering by red cell aggregates: Effect of shear rate and anisotropy. Biophysical Journal 82(4), 1696-1710 (2002)

15. Rice, J.R., White, J.S.: Norms for smoothing and estimation. Society for Industrial and Applied Mathematics Review, 243-256 (1964)

16. Shung, K.K., Smith, M.B., Tsui, B.: Principles of medical imaging. Academic Press, London (1992)

17. Lim, B., Bascom, P., Cobbold, R.: Particle and voxel approaches for simulating ultrasound backscattering from tissue. Ultrasound in Medicine and Biology 22(9), 1237-1247 (1996)

18. Shung, K., Thieme, G.: Ultrasonic scattering in biological tissues. CRC Press, Boca Raton (1993) 